\title{
Electrical Cross-Correlation Spectroscopy: Measuring Picoliter-per-Minute Flows in Nanochannels
}

\author{
Klaus Mathwig, Dileep Mampallil, Shuo Kang, and Serge G. Lemay* \\ MESA + Institute for Nanotechnology, University of Twente, PO Box 217, 7500 AE Enschede, The Netherlands
}

(Received 2 March 2012; revised manuscript received 27 June 2012; published 10 September 2012)

\begin{abstract}
We introduce all-electrical cross-correlation spectroscopy of molecular number fluctuations in nanofluidic channels. Our approach is based on a pair of nanogap electrochemical transducers located downstream from each other in the channel. When liquid is driven through this device, mesoscopic fluctuations in the local density of molecules are transported along the channel. We perform a time-offlight measurement of these fluctuations by cross-correlating current-time traces obtained at the two detectors. Thereby we are able to detect ultralow liquid flow rates below $10 \mathrm{pL} / \mathrm{min}$. This method constitutes the electrical equivalent of fluorescence cross-correlation spectroscopy.
\end{abstract}

DOI: 10.1103/PhysRevLett.109.118302

Systems in diffusive equilibrium undergo number fluctuations that encode a wealth of information on the underlying microscopic dynamics. This is explicitly exploited by methods such as fluorescence correlation spectroscopy $[1,2]$ to extract molecular properties in a wide range of biophysics experiments [3]. Here we establish a new technique to electrically probe these number fluctuations using electrodes embedded in nanochannels. We demonstrate the utility of the method by measuring ultralow liquid flow rates.

The number $N$ of solute molecules in a nanochannel volume fluctuates in time due to Brownian motion, thereby providing the basis for our measurement method. For a Poisson-distributed dilute solute, the ratio of noise to average number is $\sqrt{\left\langle(\Delta N)^{2}\right\rangle} /\langle N\rangle=1 / \sqrt{\langle N\rangle}$. Therefore, the relative size of the fluctuations is usually too small to detect in macro- or even microscale volumes; this mesoscopic effect is in this sense a hallmark of nanofluidic systems.

To probe the fluctuations, we employ electrochemical nanofluidics devices, or nanogap transducers [4], as sketched in Fig. 1(a). Electrochemically active molecules transport electrons by shuttling between closely spaced $(130 \mathrm{~nm})$, facing electrodes embedded in the walls of a nanochannel. This repeated, alternating oxidation and reduction, known as redox cycling, allows each molecule to transport several thousand electrons per second, leading to a large enhancement of the detected electrochemical current (typically several femtoamperes per molecule). For the purpose of flow detection, this device fulfills a simple function: it yields an electrical current that is directly proportional to the number of electrochemically active molecules in the volume between the electrodes as a function of time. In the $10 \mathrm{fL}$ detection volume of a device, there are about 9000000 molecules present at a $1 \mathrm{mM}$ analyte concentration (mean electrical current of $45 \mathrm{nA}$ ). Their fluctuation of 3000 molecules leads to a current noise of $15 \mathrm{pA}$, which is easily detected.
PACS numbers: 47.80.-v, 47.61.-k, 82.47.Rs, 87.64.kv

Our experimental arrangement is shown in Fig. 1. Two electrodes at the top wall of a nanofluidic channel define two distinct detection volumes. A third electrode at the bottom wall of the channel allows redox cycling to take place in both volumes. Current-time traces are recorded simultaneously at both top electrodes. When liquid is driven through the channel, the analyte molecules' average velocity is equal to the average liquid flow velocity. If the flow is fast enough to outrun the molecules' longitudinal diffusion, the number fluctuations are preserved while they travel along the channel [5]. Therefore, the same noise that is measured at the first electrode is measured again a fraction of a second later at the second electrode
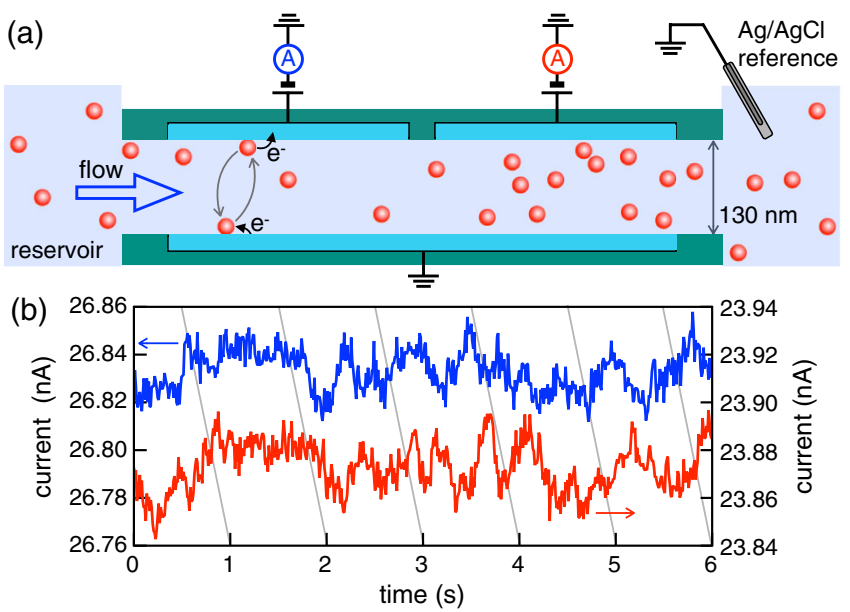

FIG. 1 (color online). (a) Schematic of the measurement concept. Fluctuations of the number of electrochemically active molecules are electrically measured at two electrodes in a nanochannel. Liquid flow velocity is determined by time-of-flight detection of these fluctuations as the molecules are transported along the channel from the upstream to the downstream transducer. (b) Raw current-time traces recorded at both electrodes at a flow rate corresponding to a time shift of approximately $0.2 \mathrm{~s}$. Gray lines are guides to the eye. 
downstream, as illustrated in Fig. 1(b). The time of flight from the center of the first electrode to the center of the second one is determined by evaluating the crosscorrelation function $G_{C}(\tau)$ of the fluctuation $\delta I(t)=$ $I(t)-\langle I\rangle$ for both current-time traces $I_{1,2}(t)$ (normalized to $G_{C}=1$ for complete correlation),

$$
G_{C}(\tau)=\left\langle\delta I_{1}(t) \delta I_{2}(t+\tau)\right\rangle /\left\langle\delta I_{1,2}^{2}\right\rangle .
$$

$G_{C}(\tau)$ peaks at the time of flight, which is in a first approximation inversely proportional to the average liquid velocity.

Nanogap sensors were fabricated as described previously $[4,6]$. In short, the devices consist of a photolithographically defined $50 \mu \mathrm{m}$ to $100 \mu \mathrm{m}$ long, $5 \mu \mathrm{m}$ wide and $130 \mathrm{~nm}$ high channel in silicon nitride fabricated on an oxidized silicon wafer. The channel volume was defined by a sacrificial $\mathrm{Cr}$ layer deposited by electron-beam evaporation that was wet etched directly before the experiment. A $3 \mu \mathrm{m}$ wide Pt electrode was positioned on the channel floor, and two $5 \mu \mathrm{m}$ wide and $24 \mu \mathrm{m}$ to $49 \mu \mathrm{m}$ long Pt electrodes separated by a $2 \mu \mathrm{m}$ wide gap formed the channel ceiling. At both ends, access holes were dry etched through the silicon nitride passivation layer to open up the channel.

We chose a syringe pump (Pump 11 Pico Plus Elite, Harvard Apparatus) to drive liquid flow, because it is the most simple and direct way for the generation of very stable flows. It further requires only a minimum of fluid handling of small sample volumes, which can thus be well protected from the environment. However, low rates of picoliters per minute are too small to be delivered-it would take several years for a drop of water $(30 \mu \mathrm{L})$ to pass through the nanochannel. Therefore we used the pump in a parallel flow control configuration $[7,8]$. Here an additional fluidic layer of microchannels in polydimethylsiloxane (PDMS) was bonded to the nanofluidic chip, so that a $100 \mu \mathrm{m}$ long microchannel with a $3 \mu \mathrm{m} \times 5 \mu \mathrm{m}$ cross section ran in parallel to the nanochannel, as shown in Fig. 2.

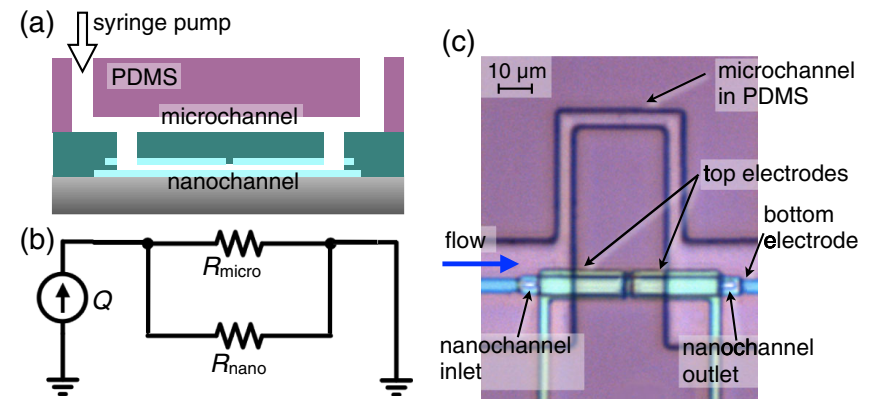

FIG. 2 (color online). (a) Schematic cross section of a nanofluidic device connected to a microchannel in a parallel flow control setup. (b) Equivalent fluidic circuit of the configuration. (c) Top view micrograph of a $50 \mu \mathrm{m}$ long device bonded to a $100 \mu \mathrm{m}$ long microchannel in PDMS.
For a Poiseuille flow in this parallel configuration, the ratio of both channels' flow rates $Q$ scales as their hydraulic resistances $R[9,10]$,

$$
\frac{Q_{\text {micro }}}{Q_{\text {nano }}}=\frac{R_{\text {nano }}}{R_{\text {micro }}} \approx \frac{\left(1-0.630 h_{m} / w_{m}\right)}{\left(1-0.630 h_{n} / w_{n}\right)} \frac{h_{m}^{3} w_{m}}{h_{n}^{3} w_{n}} \frac{L_{n}}{L_{m}},
$$

with $L_{n, m}, w_{n, m}$ and $h_{n, m}$ being the nano- and microchannel's length, width and height, respectively. Due to the cubic dependence of flow on the channel height, a high ratio of approximately 8000:1 is achieved for a $100 \mu \mathrm{m}$ long device. In practice, two devices, each with a microchannel, are connected in parallel; therefore, the nanochannel flow rate is reduced by a factor of 16000 compared to the syringe flow [11].

Ferrocenedimethanol $\left[\mathrm{Fc}(\mathrm{MeOH})_{2}\right]$, purchased from Acros, with diffusion coefficient $D=6.7 \times 10^{-10} \mathrm{~m}^{2} / \mathrm{s}$, was chosen as a redox-active species. A $1 \mathrm{mM}$ solution was prepared in milli-Q water with $1 \mathrm{M} \mathrm{KCl}$ (Sigma-Aldrich) added as background electrolyte together with $5 \mathrm{mM}$ $\mathrm{H}_{2} \mathrm{SO}_{4}$ (Sigma-Aldrich) to prevent electrode degradation. Prior to measurements, the microchannels were filled with chromium etchant (Selectipur from BASF) to remove the $\mathrm{Cr}$ sacrificial layer and release the nanogap devices. The etchant was then replaced with $5 \mathrm{mM} \mathrm{H}_{2} \mathrm{SO}_{4}$ and subsequently with the $\mathrm{Fc}(\mathrm{MeOH})_{2}$-containing solution, which was then driven with varying pump flow rates of up to $50 \mu \mathrm{L} / \mathrm{h}$.

Both top electrodes of the devices were connected to Keithley 6430 sub-femtoamp remote source meters. The two top electrodes were biased at an oxidizing potential of $0.4 \mathrm{~V}$ while the bottom electrode was short-circuited to a $\mathrm{Ag} / \mathrm{AgCl}$ reference electrode connected by tubing downstream of the device. The whole setup was shielded in a Faraday cage. Current-time traces of $25 \mathrm{~s}$ with $10 \mathrm{~ms}$ sampling intervals were simultaneously recorded at the top electrodes, as illustrated in Fig. 1(b). High-pass filtering was applied to remove low-frequency $(<50 \mathrm{mHz})$ drift.

Cross-correlation functions of the traces were then determined for different pump flow rates, as shown in Fig. 3(a). These curves exhibit clear peaks at $\tau_{\text {peak }}$, which shift to shorter times with faster flow such that $\tau_{\text {peak }} \propto$ (pump flow rate) ${ }^{-1}$. This is expected since the molecules sample the cross section homogenously and Taylor dispersion is negligible at low Péclet numbers (Pé $<0.1$ ); flow is laminar at Reynolds numbers $\operatorname{Re}<10^{-4}$ and electrokinetic effects are insignificant [11]. The decreased peak height and broadening of the peaks at low flow rates corresponds to a loss of correlation due to a more dominant influence of diffusion on the molecules' movement. This is straightforward to understand: for a pronounced peak to appear, the longitudinal rms fluctuation $\Delta x$ of the molecules during the time of flight should be smaller than the center-to-center distance $L+g$ of the electrodes, 

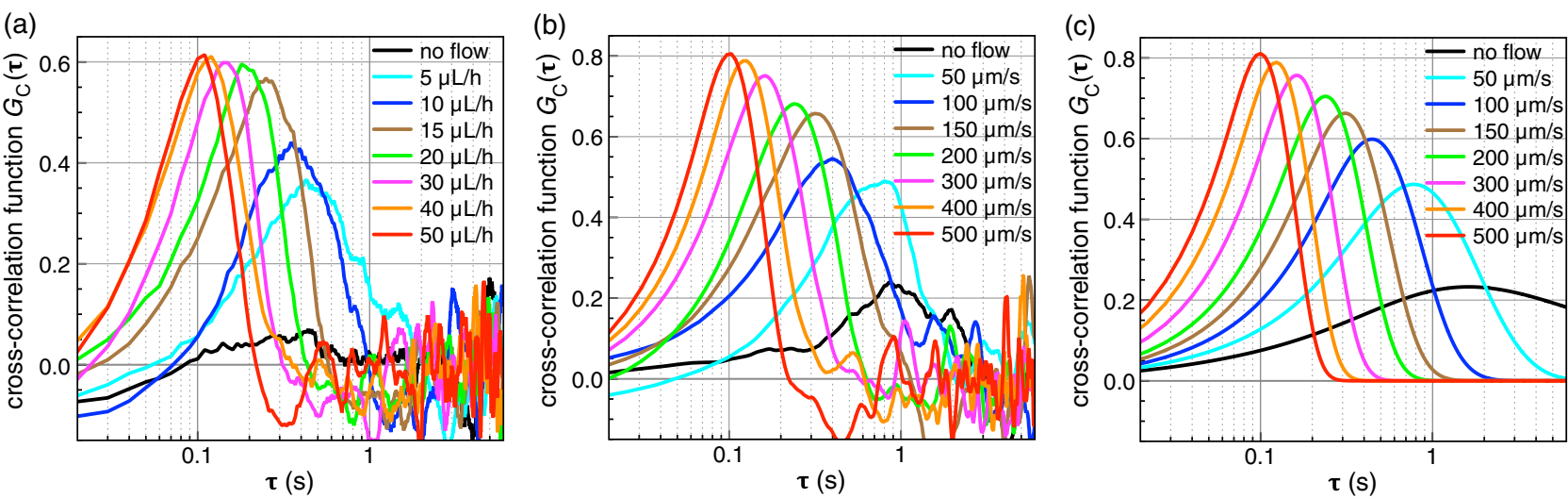

FIG. 3 (color online). (a) Cross-correlation functions of current-time traces recorded at the two $49 \mu \mathrm{m}$ long top electrodes of a $100 \mu \mathrm{m}$ long device for different pump flow rates. (b) Cross correlation of current-time traces generated by a one-dimensional random walk superimposed with flow for the same device geometry and diffusion coefficient. (c) Analytically derived cross-correlation functions for the same one-dimensional geometry and diffusion coefficient.

$\Delta x=\sqrt{2 D \tau_{\text {peak }}} \ll L+g$. This leads to the condition $v \gg 2 D /(L+g)$ for well-defined correlation peaks.

To test our hypothesis that the measured cross correlations can be understood simply as the superposition of longitudinal drift and diffusion, we performed a onedimensional random walk simulation [12]. In short, 10000 molecules were randomly distributed and underwent random steps at $1 \mu \mathrm{s}$ intervals. An additional drift term was superimposed to represent the flow velocity $v$. At each time step, the number of molecules, or occupancy, in two regions of length $L=49 \mu \mathrm{m}$ separated by a gap of $g=2 \mu \mathrm{m}$ was evaluated in a $2 \mathrm{~mm}$ long geometry with closed-loop boundary conditions. Thirty seconds long occupancy traces were generated and then cross correlated in the same way as the experimentally obtained currents. The resulting cross-correlation functions for different flow velocities are shown in Fig. 3(b). They show good agreement with the experimental data, for example exhibiting similar noise at long times $\tau$ caused by the finite duration of the traces. We attribute the slightly smaller peak height of the experimental data $(\sim 0.6$ instead of 0.8$)$ to asymmetrically fabricated top electrode areas.

To facilitate further analysis, we derived an analytical form of the cross-correlation function $G_{C}(L, g, D, v, \tau)$. In close analogy to two-beam cross-correlation spectroscopy [13], $G_{C}$ was evaluated from the expression

$$
G_{C}(\tau)=\frac{\int W_{1}(x) W_{2}\left(x^{\prime}\right) f\left(x, x^{\prime}, \tau\right) d x d x^{\prime}}{\langle N\rangle^{2} \int W_{1}(x) d x \int W_{2}\left(x^{\prime}\right) d x^{\prime}}
$$

where $W_{1,2}(x)$ correspond to the detection regions defined by top electrodes and $f$ is a number concentration correlation function, i.e., in essence the probability to find a molecule originally at the longitudinal position $x$ a time $\tau$ later at $x^{\prime}$. The details of the calculation are given in the Supplemental Material [11]. The resulting crosscorrelation function is explicitly given by

$$
\begin{aligned}
G_{C}(\tau)= & \sqrt{\frac{D \tau}{\pi L^{2}}}\left\{\exp \left[-\frac{(2 L+g-v \tau)^{2}}{4 D \tau}\right]+\exp \left[-\frac{(g-v \tau)^{2}}{4 D \tau}\right]-2 \exp \left[-\frac{(L+g-v \tau)^{2}}{4 D \tau}\right]\right\} \\
& +\frac{2 L+g-v \tau}{2 L} \operatorname{erf}\left(\frac{2 L+g-v \tau}{\sqrt{4 D \tau}}\right)+\frac{g-v \tau}{2 L} \operatorname{erf}\left(\frac{g-v \tau}{\sqrt{4 D \tau}}\right)-\frac{L+g-v \tau}{L} \operatorname{erf}\left(\frac{L+g-v \tau}{\sqrt{4 D \tau}}\right) .
\end{aligned}
$$

$G_{C}(\tau)$ is shown in Fig. 3(c): it resembles a Gaussian shape, in good agreement with the experimental as well as simulated data. The velocity necessary to outrun diffusion is given by $v \geq 4 D /(L+g)$ for $G_{C}(\tau) \geq 0.5$, consistent with our earlier analysis.

The effect of flow on correlation is further illustrated by looking at the simpler case of only one detection volume, i.e., by autocorrelation analysis. In Fig. 4, the autocorrelation functions of the same traces used for cross-correlation analysis [Fig. 3(a)] are shown as well as analytically derived functions [11]. Similar to fluorescence correlation spectroscopy, the functions exhibit a simple shape consisting of a plateau for short times (not fully captured at the limited measurement bandwidth) and an exponential $\tau^{-1 / 2}$ tail of the correlation which shifts to shorter times with increasing flow. Extrapolating the functions linearly to $G_{\mathrm{A}}=0$ leads to a time indicating the loss of correlation which is consistent with $\tau_{\text {peak }}$ (for our case $L \approx g+L$ ), as shown in Fig. 4(a). 

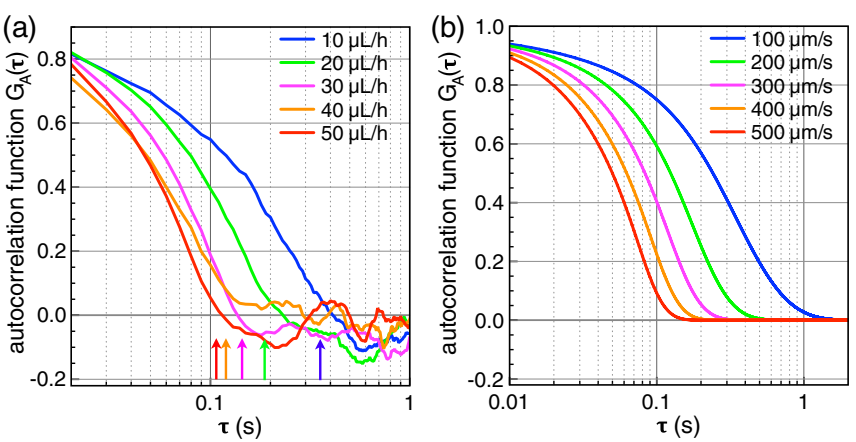

FIG. 4 (color online). (a) Autocorrelation functions determined of the same traces (but only from one electrode) used for the cross-correlation analysis. The arrows indicate the corresponding cross-correlation peak times shown in Fig. 3(a). (Functions for smaller flow rates are omitted because the short duration of the traces leads to excessive scatter.) (b) Analytically derived one-dimensional autocorrelation functions.

As a simple illustrative application, we extract the liquid flow rate from the cross correlation. In a first approximation this can be done using the time-of-flight expression $Q_{\text {nano }}=(L+g) h_{n} w_{n} / \tau_{\text {peak }}$. Nanochannel flow rates obtained in this manner are shown in Fig. 5 as a function of the syringe pump flow rate (blue circles). The molecules' fast shuttling eliminates the need for velocity corrections as used in micro particle image velocimetry [14]. Two effects need to be corrected for an accurate flow rate determination, however. First, the peak time $\tau_{\text {peak }}$ deviates up to $10 \%$ from the time-of-flight approximation for slow flow rates because the peak shape is then strongly influenced by diffusion. This effect is easily corrected by using the full analytical cross-correlation function, Eq. (4), as discussed in the Supplemental Material [11]. A second, more important source of a systematic error is reversible adsorption of redox-active molecules at the channel walls and electrodes inherent to the high surface-to-volume ratio in nanogap devices $[6,15]$. The fraction of adsorption $N_{\text {ads }} / N_{\text {tot }}$ and thereby the retardation of the analyte molecules can be readily extracted from the mesoscopic fluctuation using the expression $N_{\text {ads }} / N_{\text {tot }}=1-$ $h^{2}\left\langle\delta I^{2}\right\rangle / e D\langle I\rangle[15]$. By averaging over thirty time traces we determine a reduction of the molecule's speed to be $N_{\text {ads }} / N_{\text {tot }}=35 \% \pm 5 \%$.

Flow rate data adjusted for both adsorption and the diffusion-induced peak shift are shown as black dots in Fig. 5. We attribute the sublinear increase of the nanochannel flow at high pump flow rates to bulging of the microchannel (at a pressure drop of approximately 0.5 bar), an effect that is well known for soft PDMS microchannels $[16,17]$.

At the lowest pump rates, we measure nanochannel flow below $10 \mathrm{pL} / \mathrm{min}$, which is below the lowest previously reported value of $\sim 30 \mathrm{pL} / \min [7]$.

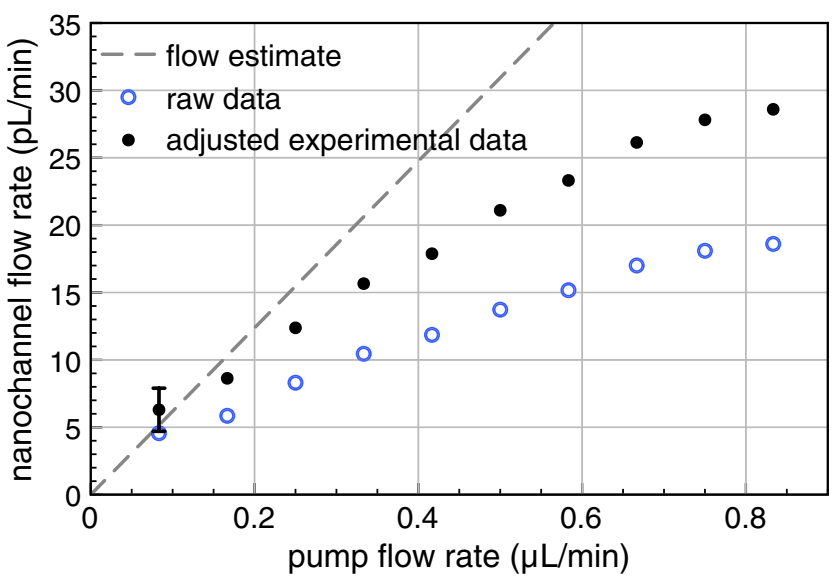

FIG. 5 (color online). Nanofluidic flow rates in a nanofluidic device as a function of syringe pump flow rate. The adjusted experimental data points (black dots) include a correction for adsorption as well as for the shift of the peak times $\tau_{\text {peak }}$. The dashed line's slope is identical to the ratio of hydraulic resistances $2 R_{\text {micro }} / R_{\text {nano }}=1 / 16000$.

While the measurements shown here were performed at a relatively high concentration of $1 \mathrm{mM}$, the method is in principle applicable at arbitrarily low concentrations because other intrinsic sources of noise exhibit the same scaling with concentration (or, equivalently, average current) but with a smaller amplitude [18]. In particular, at a bandwidth of $10 \mathrm{~Hz}$ the rms shot noise and Johnson noise are $\sqrt{2 e \times 10 \mathrm{~Hz}} \sqrt{I}=1.8 \times 10^{-9} \mathrm{~A}^{1 / 2} \sqrt{I}$ and $\sqrt{4 k T \times 10 \mathrm{~Hz} / 0.4 \mathrm{~V}} \sqrt{I}=6.4 \times 10^{-10} \mathrm{~A}^{1 / 2} \sqrt{I}$, respectively, whereas the diffusion noise dominates with $\sqrt{e D / h^{2}} \sqrt{I} \approx 10^{-7} \mathrm{~A}^{1 / 2} \sqrt{I}$.

At sufficiently low concentrations, the sensitivity becomes limited by extrinsic instrumental noise instead. A single molecule yields a current of $i=e D \epsilon / h^{2}$ with $\epsilon=$ $1-N_{\text {ads }} / N_{\text {tot }}$, and single molecule detection was previously demonstrated in $70 \mathrm{~nm}$ high diffusively coupled nanogap sensors at $i=20 \mathrm{fA}$ with a signal-to-noise ratio of 1 and an instrumental response time of $100 \mathrm{~ms}$ [12]. If the signal-to-noise ratio could be increased, e.g., by improved instrumentation or reduced channel height, it would then become possible to distinguish single molecules according to their diffusion coefficient, size or charge transfer, or by their electrochemical properties when biasing successive transducers at different redox potentials.

In conclusion, we have developed electrochemical cross-correlation spectroscopy as a new technique to study the transport of mesoscopic numbers of electroactive analyte molecules in nanofluidic volumes. Here we employed this method for the determination of record-low liquid flow rates. We also envision a broader range of applications: similar to its direct optical analogue, fluorescence (cross-) correlation spectroscopy $[13,19,20]$, the technique can be used to investigate local concentration, adsorptivity and 
chemical reaction kinetics of analyte molecules. As compared to fluorescence correlation spectroscopy, our method permits studying the properties of a different class of molecules or molecular labels (electrochemically active instead of fluorescent). Furthermore, all-electrical detection without the need for a microscope facilitates integration in microfluidic lab-on-a-chip systems, where multiple detectors in more complex nanochannel networks can also be realized.

We thank Z. Zhu and W. Sparreboom for advice about microfluidic PDMS interconnects and gratefully acknowledge financial support from the Netherlands Organization for Scientific Research (NWO) and the European Research Council (ERC).

*s.g.lemay@utwente.nl

[1] D. Magde, E. Elson, and W. W. Webb, Phys. Rev. Lett. 29, 705 (1972)

[2] E. L. Elson and D. Magde, Biopolymers 13, 1 (1974).

[3] E. L. Elson and D. Magde, Biophys. J. 101, 2855 (2011).

[4] M. A. G. Zevenbergen, B. L. Wolfrum, E. D. Goluch, P. S. Singh, and S. G. Lemay, J. Am. Chem. Soc. 131, 11471 (2009).

[5] Note that there is a strong separation of time scales: due to the high aspect ratio (700:1) geometry of the channel, a molecule crosses the channel vertically within microseconds, but it takes seconds before the longitudinal fluctuation correlation is lost completely.

[6] S. Kang, K. Mathwig, and S. G. Lemay, Lab Chip 12, 1262 (2012).
[7] H. Liang, W. J. Nam, and S. J. Fonash, in Technical Proceedings of the NSTI Nanotechnology Conference, Boston, 2008 (CRC, Boca Raton, 2008), Vol. 3, p. 281.

[8] H. Liang, Ph.D. thesis, The Pennsylvania State University, 2008.

[9] H. Bruus, Lab Chip 11, 3742 (2011).

[10] L. Rassaei, K. Mathwig, E. D. Goluch, and S. G. Lemay, J. Phys. Chem. C 116, 10913 (2012).

[11] See Supplemental Material at http://link.aps.org/ supplemental/10.1103/PhysRevLett.109.118302 for derivation and discussion of an analytical expression for a one-dimensional electrochemical auto- as well as crosscorrelation function with flow; overlay of experimentally obtained, simulated, and analytical cross-correlation functions; estimation of electroosmotic backflow in nanochannels; and details on the overall microfluidic circuitry.

[12] M. A. G. Zevenbergen, P.S. Singh, E. D. Goluch, B. L. Wolfrum, and S. G. Lemay, Nano Lett. 11, 2881 (2011).

[13] M. Brinkmeier, K. Dörre, J. Stephan, and M. Eigen, Anal. Chem. 71, 609 (1999).

[14] A. Kloostermann, C. Poelma, and J. Westerweel, Exp. Fluids 50, 1587 (2010).

[15] P. S. Singh, H.-S. M. Chan, S. Kang, and S. G. Lemay, J. Am. Chem. Soc. 133, 18289 (2011).

[16] M. A. Holden, S. Kumar, A. Beskok, and P. S. Cremer, J. Micromech. Microeng. 13, 412 (2003).

[17] T. Gervais, J. El-Ali, A. Günther, and K. F. Jensen, Lab Chip 6, 500 (2006).

[18] P. Horowitz and W. Hill, The Art of Electronics (Cambridge University Press, Cambridge, England, 1989).

[19] D. Magde, W. W. Webb, and E. L. Elson, Biopolymers 17, 361 (1978).

[20] P.S. Dittrich and P. Schwille, Anal. Chem. 74, 4472 (2002). 